\title{
MR Features of Juxta-Articular Venous Malformations of the Knee to Predict the Clinical Outcome of Sclerotherapy
}

Gnannt, Ralph ; Guggenberger, Roman ; Mueller, Daniel A ; Neuhaus, Kathrin ; Aufdenblatten, Christoph ; Theiler, Martin ; Kellenberger, Christian J ; Pfammatter, Thomas

\begin{abstract}
PURPOSE To analyze and correlate preinterventional magnetic resonance (MR) imaging findings with clinical symptoms after percutaneous sclerotherapy of venous malformations (VMs) adjacent to the knee. MATERIALS AND METHODS Twenty-five patients (mean age, 24 y; range, 7-55 y; 11 female) with $26 \mathrm{VMs}$ adjacent to the knee undergoing sclerotherapy (direct puncture, diagnostic angiography, sclerosant injection) were identified, and MR imaging findings were analyzed. The VM involved the synovium of the knee joint in 19 of 26 cases (76\%). These lesions were associated with joint effusion ( 3 of $19 ; 16 \%$ ), hemarthrosis (4 of $19 ; 21 \%$ ), or synovial thickening (16 of $19 ; 84 \%$ ). Follow-up ended 6-8 weeks after the first or second sclerotherapy session if complete pain relief was achieved or 3 months after the third sclerotherapy session. Treatment outcomes were categorized as symptom improvement (complete or partial pain relief) or poor response (unchanged or increased pain). RESULTS Forty-nine percutaneous sclerotherapy sessions were performed. Despite the absence of signs of knee osteoarthritis, patients with a VM involving the synovium (8 of $14 ; 57 \%$ ) showed a poor response to sclerotherapy (1 of 8 [13\%] pain-free after 1 sclerotherapy session). Among patients with VMs with no associated joint alteration and no synovial involvement (6 of 14;43\%), 5 of $6(83 \%)$ showed improvement of symptoms after 1 sclerotherapy session $(\mathrm{P}<.05)$. CONCLUSIONS Juxta-articular VMs of the knee are frequently associated with hemarthrosis and synovial thickening. Patients with signs of osteoarthritis and synovial involvement of the VM on presclerotherapy MR imaging deserve special consideration, as these findings predict worse clinical symptoms after sclerotherapy.
\end{abstract}

DOI: https://doi.org/10.1016/j.jvir.2019.11.014

Posted at the Zurich Open Repository and Archive, University of Zurich

ZORA URL: https://doi.org/10.5167/uzh-179916

Journal Article

Accepted Version

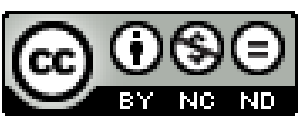

The following work is licensed under a Creative Commons: Attribution-NonCommercial-NoDerivatives 4.0 International (CC BY-NC-ND 4.0) License.

Originally published at:

Gnannt, Ralph; Guggenberger, Roman; Mueller, Daniel A; Neuhaus, Kathrin; Aufdenblatten, Christoph; Theiler, Martin; Kellenberger, Christian J; Pfammatter, Thomas (2020). MR Features of Juxta-Articular 
Venous Malformations of the Knee to Predict the Clinical Outcome of Sclerotherapy. Journal of Vascular and Interventional Radiology, 31(4):551-557.

DOI: https://doi.org/10.1016/j.jvir.2019.11.014 


\title{
Magnetic Resonance Features of Juxta Articular Venous Malformations of the Knee to Predict the Clinical Outcome of Sclerotherapy
}

\author{
R. Gnannt ${ }^{1,2}$, R. Guggenberger ${ }^{1}$,D. A. Müller ${ }^{3}$, K. Neuhaus ${ }^{4}$, Ch. Aufdenblatten ${ }^{5}$, \\ M. Theiler ${ }^{6}$, C.J. Kellenberger ${ }^{2}$, T. Pfammatter ${ }^{1}$ \\ ${ }^{1}$ Department of Diagnostic and Interventional Radiology, University Hospital Zurich, Zurich, Switzerland \\ ${ }^{2}$ Diagnostic Imaging, University Children's Hospital Zurich, Zurich, Switzeriand \\ ${ }^{3}$ Orthopaedics, Der Balgrist - Universitätsklinik, Zurich, Switzerland \\ ${ }^{4}$ Division of Plastic and Reconstructive Surgery, Department of Surgery, University Children's Hospital Zurich, \\ Zurich, Switzerland \\ ${ }^{5}$ Orthopaedics, Department of Surgery, University Children's Hospital Zurich, Zurich, Switzerland \\ ${ }^{6}$ Division of Pediatric Dermatology, University Children's Hospital Zurich, Zurich, Switzerland \\ Type of Manuscript: \\ Journal: \\ Original research \\ Journal of Vascular and Interventional Radiology
}

Key Words:

Conflict of Interest:

Corresponding Author: knee, venous, malformation, sclerotherapy, arthroscopy

The authors declare that they have no conflict of interest.

Ralph Gnannt, MD

E-mail: ralph.gnannt@usz.ch 


\section{ABSTRACT}

\section{PURPOSE:}

To analyze and correlate pre-interventional magnetic resonance imaging (MRI) findings with clinical symptoms after percutaneous sclerotherapy treatment of venous malformations (VM) adjacent to the knee.

\section{MATERIAL AND METHODS:}

25 patients (mean age 24yrs, range 7-55yrs; 11 females) with $26 \mathrm{VMs}$ adjacent to the knee undergoing sclerotherapy (direct puncture, diagnostic angiography, sclerosant injection) were identified and MRI findings were analyzed. The VM involved the synovium of the knee joint in 19/26 (76\%) of all patients. These lesions were associated with joint effusion (3/19, $16 \%)$, hemarthrosis $(4 / 19,21 \%)$ or synovial thickening $(16 / 19,84 \%)$. Follow up period of each patient ended 6 to 8 weeks after the first or second sclerotherapy session if complete pain relief was achieved or 3 months after the third sclerotherapy session. Treatment outcome was categorized into improved symptoms (complete or partial pain relief) and poor response (unchanged or increased pain).

\section{RESULTS:}

49 percutaneous sclerotherapy sessions were performed. Despite absence of signs of knee osteoarthritis patients with a VM involving the synovium $(8 / 14,57 \%)$ responded poorly to sclerotherapy $(1 / 8,13 \%$ pain free after one sclerotherapy session). On the other hand, patients 
with VMs with no associated joint alteration and no synovial involvement $(6 / 14,43 \%)$ showed improvement of symptoms after one single sclerotherapy session in $5 / 6(83 \%)$ patients $(p<0.05)$.

\section{CONCLUSION:}

Juxta-articular VMs of the knee are frequently associated with hemarthrosis and synovial thickening. Patients with signs of osteoarthritis and synovial involvement of the VM on presclerotherapy MR deserve special consideration as these findings hamper the clinical symptoms after sclerotherapy. 


\section{INTRODUCTION:}

Venous malformations (VM) are congenital, benign vascular anomalies consisting of deformed venous channels [1]. Depending on the extension of the VM, subcutaneous tissue, muscles, tendons or even bone may be involved [2][3]. If the VM is located adjacent to the knee joint early magnetic resonance imaging (MRI) is important to evaluate the exact juxta-articular extent of the lesion and its relation to different joint structures [4]. Additionally, MRI can be correlated with clinical symptoms and guide the interventional radiologist towards the optimal treatment location [5].

Indications for invasive treatment of a VM is persistent pain and impaired mobility, after conservative treatment has failed. The mainstay of invasive therapy of VMs nowadays is percutaneous sclerotherapy [6]. A wide variety of sclerosing agent with different mechanism of action on target tissue have been described in the literature [7]. Sodium Tetradecyl Sulfate (STS), ethanol, polidocanol, and bleomycin are the ones most commonly used [8][9][10]. However, the clinical response to sclerotherapy varies among patients and is affected by anatomical location, size, extent of the VM and number of treatment sessions [11][12].

The aim of the study was to analyze pre-interventional magnetic resonance imaging and correlate the findings with the clinical symptoms of percutaneous sclerotherapy treatment of VM's adjacent to the knee joint in order to identify predictors for a favourable outcome of sclerotherapy in VMs adjacent to the knee joint. 


\section{MATERIAL \& METHODS}

Study Design:

This retrospective cohort study was conducted in a tertiary referral teaching hospital. Institutional Research Ethics Board approval was obtained. The study cohort was identified from radiology reports on consecutive patients who had a VM adjacent to the knee joint identified on MRI and at least one session of percutaneous sclerotherapy with a percutaneous angiography performed. The knee joint was defined to extend from the distal femoral physis to the proximal tibial physis. The search period started January 2008 to ensure a sufficient sample size until December 2017 to allow adequate follow-up of all patients.

Combinations of the following search words were used: magnetic resonance imaging, knee, venous malformation, vascular malformation, joint, lesion. If a patient underwent more than one MR examination, only the one prior to the sclerotherapy was reviewed. Only patients without prior treatment (percutaneous sclerotherapy, surgery) were included in the study. One patient with a Klippel-Trenauney syndrome was excluded from the study.

\section{$M R$ examination}

The standard knee protocol with contrast at our institution was performed on a 1.5-Tesla scanner (Skyra, Siemens Medical, Healthineers, Erlangen, Germany) with an eight-channel phased array coil. The sequences obtained during MRI were the following: coronal turboinversion recovery magnitude (TIRM, TR/TE $=7470 \mathrm{~ms} / 38 \mathrm{~ms}$ ), sagittal proton density weighted sequence with fat saturation (PDfs, TR/TE $=6690 \mathrm{~ms} / 38 \mathrm{~ms}$ ), axial $\mathrm{T} 1$ weighted turbos spin echo sequence $(\mathrm{T} 1 \mathrm{TSE}, \mathrm{TR} / \mathrm{TE}=449 \mathrm{~ms} / 11 \mathrm{~ms}$ ) and an axial and sagittal $\mathrm{T} 1$ fat saturated post-contrast 
sequence $(\mathrm{TR} / \mathrm{TE}=615 \mathrm{~ms} / 11 \mathrm{~ms})$ at $3 \mathrm{~mm}$ slice thickness and increment, respectively. Respective field of views were adjusted to patient anatomy and size.

Image review

First, the images were reviewed by a radiologist with 10 years of experience to ensure MR findings are compatible with the diagnosis of VM. A VM was defined as a T2 hyperintense and T1 hypointense lesion with mixed contrast uptake (due to slow flow characteristic, phleboliths and/or thrombosis) [13]. To determine the exact location of a VM, the knee joint was separated into three parts according to the study form Jans et al: the superior third extended from the femoral physis to the inferior aspect of the suprapatellar pouch (A), the inferior third from the inferior aspect of the synovium of the knee to the tibial physis (C) and the middle third between the superior and inferior third (B) [4]. The approximate VM volume was calculated by averaging the measurements of the three planes (ap, lat, cc) [14]. Then the VM and the adjacent knee joint was assessed more in detail separately by two radiologists who were blinded to the final outcome ( 9 and 13 years of experience): extent of VM, (separate from knee joint, reaching capsule or reaching synovium), presence of joint effusion, hemarthrosis, synovial thickening, and bone involvement. In case of disagreement a third reader (32 years of experience) was included and the imaging assessed in a consensus manner between the three readers.

Intraprocedural Venography and Sclerotherapy

Depending on the sclerosant utilized, the number of punctures planned and the age of the patient the procedures were performed under general anesthesia $(n=2)$, moderate sedation $(n=45$, 
local anesthesia $(n=5)$ or none of them $(n=2)$. Sonographic guided direct puncture of the VM was initially performed. Depending on the extent of the lesion at least one needle was placed into the VM. To study the intralesional needle positioning, the extension, volume and venous outflow of a VM, all patients received a phlebography by injecting contrast agent through each needle (nonionic, iso-osmolar contrast agent iodixanol $320 \mathrm{mg} / \mathrm{ml}$, Visipaque, GE Healthcare, Chalfont St. Giles, UK). We perform this as a routine procedure before injecting sclerosans. As a sclerosing agent either Sodium Tetradecyl Sulfate (Fibro-Vein ®, STD Pharmaceutical Products Ltd., Hereford, U.K., $3 \%$ mixed with room air $1: 1$ to create a foam), ethanol $96 \%$ or Aethoxysclerol 3\% (Lauromacrogol 400, Kreussler Pharma, Wiesbaden, Germany) was injected at the discretion of the investigator. The volume injected depended on the estimated volume after phlebography and the outflow of the lesion. If there was no or only partial pain relief after the first sclerotherapy session, a second treatment 6 to 8 weeks after the first one was performed and, if necessary, a third session another 6 to 8 weeks later using the same routine protocol.

\section{Follow-up period}

The follow up period of each patient ended 6 to 8 weeks after the first or second sclerotherapy session if complete pain relief was achieved or 3 months after the third sclerotherapy session disregarding whether pain relief was achieved. Clinical symptoms after treatment was extracted from the physician's note during the last follow up visit and categorized according to the subjective patient symptoms of pain into $0=$ complete pain relief, $1=$ partial pain relief, $2=$ unchanged pain, $3=$ increased pain. Category 2 and 3 were defined as poor responders.

\section{Statistical Analysis:}


For statistical analysis SPSS version 23.0 for Windows (SPSS Inc., Chicago, IL) was used. Frequency distributions and cross-tabulations of variables of interest were obtained. The Shapiro-Wilk test was used to assess normal distribution. Results were expressed as mean \pm SD or median where appropriate. Student's t-test or Mann-Whitney U test was used for group comparisons for continuous variables. Chi-square or Fisher exact test was used to compare categorical variables. Differences were considered significant at $\mathrm{p}<0.05$ (two-tailed). The interreader agreement was calculated using Cohen's kappa statistics. The inter-reader agreement was defined excellent $(\kappa>0.81)$, good $(\kappa=0.61-0.80)$, moderate $(\kappa=0.41-0.60)$, fair $(\kappa=0.21-0.40)$ and poor $(\kappa<0.20)$. 


\section{RESULTS}

Between January 2008 and December 2017, 25 patients (14 males, 11 females, mean age of patients $24 \mathrm{yrs}$, range 7-55yrs) with $26 \mathrm{VMs}$ adjacent to knee joint with an MRI prior to any invasive treatment were identified and included in the study. One patient had bilateral knee VM. One patient had only an ultrasound of a small VM adjacent to knee joint and no MRI prior to percutaneous sclerotherapy and was not included in the study cohort of a total of 25 patients.

The mean diameter of all VMs was $5.0 \mathrm{~cm}(\mathrm{SD}+1-0.75 \mathrm{~cm}$, range $3.0 \mathrm{~cm}-7.4 \mathrm{~cm}) .3$ lesions were located in the upper third, 14 the middle third, and 9 in the lower third of the knee joint. 49 percutaneous sclerotherapy sessions were performed in 26 joints (mean sclerotherapy session per patient 1.9 , range 1-3). On average 2.4 needles (range $1-5$ ) were placed into the lesion per session and used for sclerotherapy agent injection. Out of all 49 sclerotherapy sessions, Ethanol $96 \%$ was used in 51\% (25/49), Aethoxysclerol 3\% in 14\% (7/49) and STS 3\% in 35\% (17/49) as a sclerosing agent. Mean follow-up time was 351 days (range 43-1268 days).

The VM involved the synovium of the knee joint in $19 / 26(73 \%)$ of all patients $(\kappa=0.71)$. In $5 / 26(19 \%)$ the lesion was completely separate from the knee joint and in only $2 / 26(8 \%)$ the VM involved the knee capsule. In those patients where the VM involved the synovium, joint effusion was seen in $16 \%(3 / 19)$, hemarthros in $21 \%(4 / 19)$, and synovial thickening in $84 \%$ (16/19). The pre-sclerotherapy MRI showed signs of joint degeneration in 11 out of 26 cases (42\%) as defined as chondral lesions in $38 \%(10 / 26)$, subchondral sclerosis in $23 \%(6 / 26)$, and osteophytes in $15 \%(4 / 26)$. 
Regarding clinical symptoms after sclerotherapy, out of the 26 knee VM 69\% (18/26) were pain free $(54 \%, 14 / 26)$ or had less pain $(15 \%, 4 / 26)$ after the final clinical assessment after a mean of 1.6 (range 1-3) sclerotherapy sessions. $27 \%$ (7/26) patients reported pain remained unchanged and one patient $(4 \%, 1 / 26)$ experienced worsening pain after the final assessment. Therefore, in total 8 patients $(31 \%, 8 / 26)$ showed no positive response after a mean of 2.6 (range 2-3) sclerotherapy treatments of their VM (Figure 1).

Treatment response was then correlated with pre-interventional MRI findings. In those 8 patients who responded poorly to sclerotherapy, synovial involvement was shown in $88 \%(7 / 8)$, chondral lesions in $75 \%(6 / 8)$, osteophytes in 38\% (3/8) and subchondral sclerosis in $40 \%(4 / 8)$. Within the group of 18 patients with good treatment response VMs synovial involvement was seen in $67 \%(12 / 18)$, chondral lesions in $22 \%(4 / 18)$, osteophytes in $6 \%(1 / 18)$, and subchondral sclerosis in $11 \%(2 / 18)$. In the group with good treatment response only $4 / 18(22 \%)$ had signs of osteoarthritis compared to $7 / 8(88 \%)$ in the group with poor response $(\mathrm{p}<0.01)$. None of the patients showed hemarthrosis after sclerotherapy treatment during the follow up time (Table 1). Additionally, there was no significant difference in treatment outcome comparing sclerosing agents used for sclerotherapy treatment. Treatment outcome in correlation with the anatomical location, size, synovial involvement and the sclerosing agent used is summarized in Table 1.

However, patients without any signs of knee osteoarthritis on MR (regardless extent of the lesion; $15 / 26,57 \%), 6$ out of 15 patients (40\%) showed improvement of symptoms after one single sclerotherapy session. In 8 of these 15 patients the VM involved the synovium and only one out of those 8 patients $(13 \%)$ reported complete or partial pain relief after one single 
sclerotherapy session (Figure 2). Compared to the patients where the VM showed no involvement of the synovium (and no signs of osteoarthritis, 7/15), 6 out of $7(86 \%)$ reported complete or partial pain relief after one single sclerotherapy session $(\mathbf{p}<0.05)$ (Figure 3$)$. However, there was no significant difference in overall treatment outcome regardless if the synovia of the knee was involved by the VM or not $(p=0.3748$, Table 1$)$. 


\section{DISCUSSION}

VMs of the extremities may be asymptomatic and present as an incidental finding or may cause significant pain and potentially limb-threatening complications. One of the most disabling complications of VMs in the juxta articular knee area is recurrent hemarthrosis in the knee joint with consecutive cartilage destruction and long-term knee joint degenerative changes [15]. Intermittent bleeding into the joint space usually starts early in childhood and can be frequently observed in extensive VMs of the lower limb [16]. In our cohort, hemarthrosis was seen in 15\% of all patients on MR imaging although number of patients with intermittent bleeding into the knee joint might be higher.

Exposure of human cartilage tissue to blood leads to progressive damage of the joint surface and the underlying bone with consequently bad prognosis with joint degeneration similar to hemophilia. The intraarticular blood leads to iron deposition and to cartilage denudation, degenerative joint changes and finally signs of osteoarthritis [17]. These features mimic those typically encountered in haemophilic arthropathy or other causes of joint degeneration, such as inflammatory arthropathy or pigmented villonodular synovitis, which all require different management [18]. Thus, radiologists are playing an important role in correctly classifying the extent of a VM and consequently guide the multidisciplinary team that takes care of a patient with respect to therapeutic options.

This study aimed to correlate MRI findings with clinical symptoms after sclerotherapy treatment for VMs of the knee area. Of this patient cohort of 26 juxta articular VM, 19 (73\%) showed synovial involvement and out of those, 11 had signs of osteoarthritis (42\%). Although 
osteoarthritis of the knee has a high prevalence ( $9 \%$ at 20 years of age, $17 \%$ at 34 years of age), our patient cohort of VMs adjacent to the knee joint showed a higher prevalence of knee osteoarthritis (mean age 24 years) [19][20]. 8 out of the 26 (31\%) VMs showed bone involvement. Although Nakamura et al stated that absent of bone involvement in VM of the extremity is an independent predictor for satisfaction with treatment, only one (13\%) of our patient with an unfavourable outcome showed boney involvement on pre-sclerotherapy MRI [21]. These results support the importance of MRI in the evaluation of VMs and assessment of possible induced structural changes of the knee joint.

In general, first line treatment after failed conservative management of a symptomatic VM is radiology guided percutaneous sclerotherapy with symptom relief in up to $95 \%$ [22]. Compared to the literature our patient cohort with juxta articular VM of the knee showed an inferior response rate to sclerotherapy treatment $(69 \%$ of patients reported complete or partial pain relief after a mean of 1.6 sclerotherapy sessions). One of the reasons might be the proximity of the VM close to a very mobile joint in an exposed location in the human body and therefore vulnerable to minor trauma. This cohort had a high number of patients with signs of osteoarthritis and those patients responded poorly to sclerotherapy $(7 / 8,88 \%)$ compared to the ones without signs of osteoarthritis $(4 / 18,22 \%$; $<<0.01)$ on pre-sclerotherapy MRI. It seems obvious that pain is at least partially induced by the degenerative changes of the knee joint itself and not only by the juxta articular knee VM.

Even in patients without any signs of osteoarthritis ( $58 \%$ of the cohort) there was a difference in treatment outcome when comparing patients having a VM adjacent to the knee joint 
with or without synovial involvement. Only $13 \%$ of the patients with a VM involving the synovia of the knee joint reported partial or complete pain relief after one single sclerotherapy session (despite the absence of signs of osteoarthritis on pre-sclerotherapy MRI). However, $86 \%$ (6/7) of patients with no signs of osteoarthritis and no synovial involvement of the VM experienced partial or complete pain relief after one single sclerotherapy treatment $(p<0.05)$. After treatment completion (up to three sclerotherapy sessions) there was no significant difference in treatment outcome regardless synovial involvement by malformed veins $(p=0.3748)$

As previously shown surgical arthroscopy and wide synovectomy should be considered in all knee VMs involving the synovia. Dalmonte et al surgically resected 14 knee VM and all patients (mean age 5 years) were symptom free after a follow-up of 6 month [23]. He concluded, surgical excision is always indicated and should be performed as early as possible. Pireau et al highlighted the importance of early surgery in patients with intra-articular VM, even if asymptomatic to avoid hemarthrosis to protect the cartilage from further erosion [24]. In this patient cohort 8 patients with a mean age of 12.3 years underwent surgical resection of a venous malformation adjacent to the knee joint. All of these patients were suffering from knee pain and hemarthrosis and in 5 patients, chondropathy was already present. After a mean follow-up of 5.1years only one patient suffered from reoccurrence of hemarthrosis and none of the children had a substantial loss of function over time.

The data in this study suggests, that VMs adjacent to the knee joint are difficult to manage by the means of percutaneous sclerotherapy alone. VMs with synovial involvement need 
special considerations and this data at least question sclerotherapy as a first line treatment.

Another therapeutic approach is to embolize the VM adjacent to the knee joint with N-butyl-2cyanoacrylate to control bleeding and to facilitate surgical resection [25]. Uller et al embolized with this technique seven intra-articular VMs of the knee prior to resection with a follow-up time ranged between 0.2 to 4 years [26]. It remains unclear, if this measure really helps to prevent from hemarthrosis and consecutive osteoarthropathy in the long term. Further investigation is needed, if a combined therapeutic approach is better to help those patients in the long term: early synovectomy and percutaneous sclerotherapy of the residual VM.

This study has limitations. First, the sample size is small. Second, there was a variety of sclerotherapeutic agent used in this patient cohort, the agent was chosen by the interventional radiologist. The cohort showed no significant difference regarding sclerotherapeutic agent used and the clinical symptoms after sclerotherapy. Additionally, there is no prospective study which compares different sclerosing agent. Reoccurrence of pain after sclerotherapy of VMs is a very well known problem. During our relatively short follow-up time, there was no relapse reported in the patient history. However, this study focusses on MRI findings of juxta articular VMs and correlate those with the clinical symptoms after sclerotherapy treatment. Third, we did not correlate clinical with imaging outcome since we don't routinely perform MR after sclerotherapy treatment. Caty et al found a tendency of higher contrast uptake at 10 minutes post contrast in patients who responded to percutaneous sclerotherapy treatment. However, other studies demonstrated, that clinical outcome after sclerotherapy treatment of VMs do not necessarily correlate with imaging outcome [27][28]. 
In conclusion, symptomatic juxta articular VM of the knee joint are frequently associated with hemarthrosis and synovial thickening. Patients with signs of osteoarthritis and synovial involvement of the VM on pre-sclerotherapy MR deserve special consideration as these findings hamper the clinical symptoms after sclerotherapy. An additional assessment of the affected knee joint by an orthopaedist who is familiar with vascular anomalies before the first sclerotherapy treatment is recommended. 


\section{REFERENCES}

[1] J. B. Mulliken and J. Glowacki, "Hemangiomas and vascular malformations in infants and children: A classification based on endothelial characteristics," Plast. Reconstr. Surg., vol. 69(3), pp. 412-420, 1982.

[2] L. F. Donnelly, D. M. Adams, and G. S. Bisset, "Vascular malformations and hemangiomas: A practical approach in a multidisciplinary clinic," Am. J. Roentgenol., vol. 174(3), pp. 597-608, 2000.

[3] C. C. Breugem, M. Maas, J. A. Reekers, and C. M. A. M. Van der Horst, "Use of magnetic resonance imaging for the evaluation of vascular malformations of the lower extremity," Plast. Reconstr. Surg., vol. 108(4), pp. 870-7, 2001.

[4] L. Jans, M. Ditchfield, J. L. Jaremko, N. Stephens, and K. Verstraete, "MRI demonstrates the extension of juxta-articular venous malformation of the knee and correlates with joint changes.," Eur. Radiol., vol. 20, no. 7, pp. 1792-1798, Jul. 2010.

[5] L. M. Fayad, L. Fayad, T. Hazirolan, D. Bluemke, and S. Mitchell, "Vascular malformations in the extremities: emphasis on MR imaging features that guide treatment options.," Skeletal Radiol., vol. 35, no. 3, pp. 127-137, Mar. 2006.

[6] Y. Yao, N. N. T. Lomis, S. M. Scott, H. C. Yoon, and F. J. Miller, "Percutaneous sclerotherapy for congenital venous malformations in the extremities," Orthopedics, vol. 24(1), pp. 45-51, 2001.

[7] C. J. M. Van Der Vleuten, A. Kater, M. H. W. A. Wijnen, L. J. Schultze Kool, and M. M. Rovers, "Effectiveness of sclerotherapy, surgery, and laser therapy in patients with venous 
malformations: A systematic review," Cardiovasc. Intervent. Radiol., vol. 37(4), pp. $977-$ $89,2014$.

[8] P. E. Burrows and K. P. Mason, "Percutaneous Treatment of Low Flow Vascular Malformations," J. Vasc. Interv. Radiol., vol. 15, no. 5, pp. 431-445, May 2004.

[9] J. C. O’Donovan, J. S. Donaldson, F. P. Morello, J. M. Pensler, R. L. Vogelzang, and B. Bauer, "Symptomatic hemangiomas and venous malformations in infants, children, and young adults: Treatment with percutaneous injection of sodium tetradecyl sulfate," Am.J. Roentgenol., vol. 169(3), pp. 723-9, 1997.

[10] G. M. Legiehn and M. K. S. Heran, “A step-by-step practical approach to imaging diagnosis and interventional radiologic therapy in vascular malformations," Semin. Intervent. Radiol., vol. 27(2), pp. 209-31, 2010.

[11] M. Goyal, P. A. Causer, and D. Armstrong, "Venous vascular malformations in pediatric patients: Comparison of results of alcohol sclerotherapy with proposed MR imaging classification," Radiology, vol. 223(3), pp. 639-44, 2002.

[12] W. S. Yun et al., "Predictors of response to percutaneous ethanol sclerotherapy (PES) in patients with venous malformations: Analysis of patient self-assessment and imaging," $J$. Vasc. Surg., vol. 50(3), pp. 581-9, 2009.

[13] S. Puig, H. Aref, V. Chigot, B. Bonin, and F. Brunelle, "Classification of venous malformations in children and implications for sclerotherapy.," Pediatr. Radiol., vol. 33, no. 2, pp. 99-103, Feb. 2003.

[14] V. Caty et al., "Clinical validation of semi-automated software for volumetric and dynamic contrast enhancement analysis of soft tissue venous malformations on Magnetic Resonance Imaging examination," Eur. Radiol., vol. 24(2), pp. 542-51, 2014. 
[15] C. C. Breugem, M. Maas, S. J. M. Breugem, G. R. Schaap, and C. M. A. M. van der Horst, "Vascular malformations of the lower limb with osseous involvement," J. Bone Jt. Surg. - Ser. B, vol. 85(3), pp. 399-405, 2003.

[16] O. Enjolras, D. Ciabrini, E. Mazoyer, C. Laurian, and D. Herbreteau, "Extensive pure venous malformations in the upper or lower limb: A review of 27 cases," J. Am. Acad. Dermatol., vol. 36(2 Pt 1), pp. 219-25, 1997.

[17] N. W. D. Jansen, G. Roosendaal, J. W. J. Bijlsma, J. DeGroot, and F. P. J. G. Lafeber, "Exposure of human cartilage tissue to low concentrations of blood for a short period of time leads to prolonged cartilage damage: An in vitro study," Arthritis Rheum., vol. 56(1), pp. 199-207, 2007.

[18] H. Madani et al., "Peripheral limb vascular malformations: An update of appropriate imaging and treatment options of a challenging condition," British Journal of Radiology. p. 88(1047), 2015.

[19] F. D. Anderson JJ, "No TitleFactors associated with osteoarthritis of the knee in the first national Health and Nutrition Examination Survey (HANES I). Evidence for an association with overweight, race, and physiacl demands of work," Am J Epidemiol, vol. 128(1), pp. 179-89, 1988.

[20] M. R. Felson DT, Naimark A, Anderson J, Kazis L, Castelli W, "No TitleThe prevalence of knee osteoarthritis in the elderly. The Framingham Osteoarthritis Study," Arthritis Rheum, vol. Aug, no. 30(8), pp. 914-8, 1987.

[21] M. Nakamura et al., "Percutaneous sclerotherapy for venous malformations in the extremities: clinical outcomes and predictors of patient satisfaction," J. Korean Phys. Soc., vol. 3:520, 2014. 
[22] W. N. Orlando JL, Caldas JG, Campos HG, Nishinari K, "Ethanol Sclerotherapy of Superficial Venous Malformation: a New Procedure.," Dermatology, vol. 220, no. 4, pp. $376-380,2010$.

[23] P. Dalmonte, C. Granata, E. Fulcheri, N. Vercellino, S. Gregorio, and G. Magnano, "Intraarticular venous malformations of the knee," J. Pediatr. Orthop., vol. 32, no. 4, pp. 394 $398,2012$.

[24] N. Pireau, L. M. Boon, P. Poilvache, and P.-L. Docquier, "Surgical Treatment of Intraarticular Knee Venous Malformations: When and How?," J. Pediatr. Orthop., vol. 36, no. 3, pp. 316-322, Apr. 2016.

[25] P. E. Burrows and K. P. Mason, "Percutaneous Treatment of Low Flow Vascular Malformations," Journal of Vascular and Interventional Radiology. pp. 15(5), 431-45, 2004.

[26] W. Uller et al., "Preoperative Embolization of Venous Malformations Using n-Butyl Cyanoacrylate," Vasc. Endovascular Surg., vol. 52(4), pp. 269-274, 2018.

[27] J. Spence, T. Krings, K. G. TerBrugge, L. B. Da Costa, and R. Agid, "Percutaneous sclerotherapy for facial venous malformations: Subjective clinical and objective MR imaging follow-up results," Am. J. Neuroradiol., vol. 31(5), pp. 955-60, 2010.

[28] K. T. Tan, J. Kirby, D. K. Rajan, E. Hayeems, J. R. Beecroft, and M. E. Simons, "Percutaneous Sodium Tetradecyl Sulfate Sclerotherapy for Peripheral Venous Vascular Malformations: A Single-Center Experience," J. Vasc. Interv. Radiol., vol. 18(3), pp. 343$51,2007$. 


\section{TABLES}

Table 1:

Flow Chart of the clinical outcome of the patient cohort. Patients had less or were free of pain after a mean of 1.6 (range 1-3) sclerotherapy sessions. The poor responders had a mean of 2.6 (range 2-3) sclerotherapy sessions.

Table 1:

Clinical symptoms in correlation with age, VM diameter \& anatomical location, MR features, hemarthrosis, and sclerotherapeutic agent used. * range in brackets.

Anatomical location of the VM: superior third extended from the femoral physis to the inferior aspect of the suprapatellar pouch (A), middle third between the superior and inferior third (B) inferior third from the inferior aspect of the synovium of the knee to the tibial physis (C)

\section{FIGURES}

Figure 1:

Flow Chart of the clinical outcome of the patient cohort. Patients had less or were free of pain after a mean of 1.6 (range 1-3) sclerotherapy sessions. The poor responders had a mean of 2.6 (range 2-3) sclerotherapy sessions.

\section{Figure 2:}

(a) Sagittal T2 weighted fat saturated image of the knee of a 33 years old male patient. Patient had an extensive VM of the knee with synovial involvement (arrows). (b) \& (c) Despite there were no signs of osteoarthritis on pre-treatment MR patient was still symptomatic after three sclerotherapy sessions.

\section{Figure 3:}

(a) \& (b) Sagittal and coronal T2 weighted, fat saturated images of the knee of a 12 year old male with a venous malformation without involvement of the synovia and no signs of osteoarthritis. (c) \& (d) After ultrasound guided needle placement angiography was perfomed which shows the extent of the VM. Patient was pain free after one sclerotherapy session with STS. 


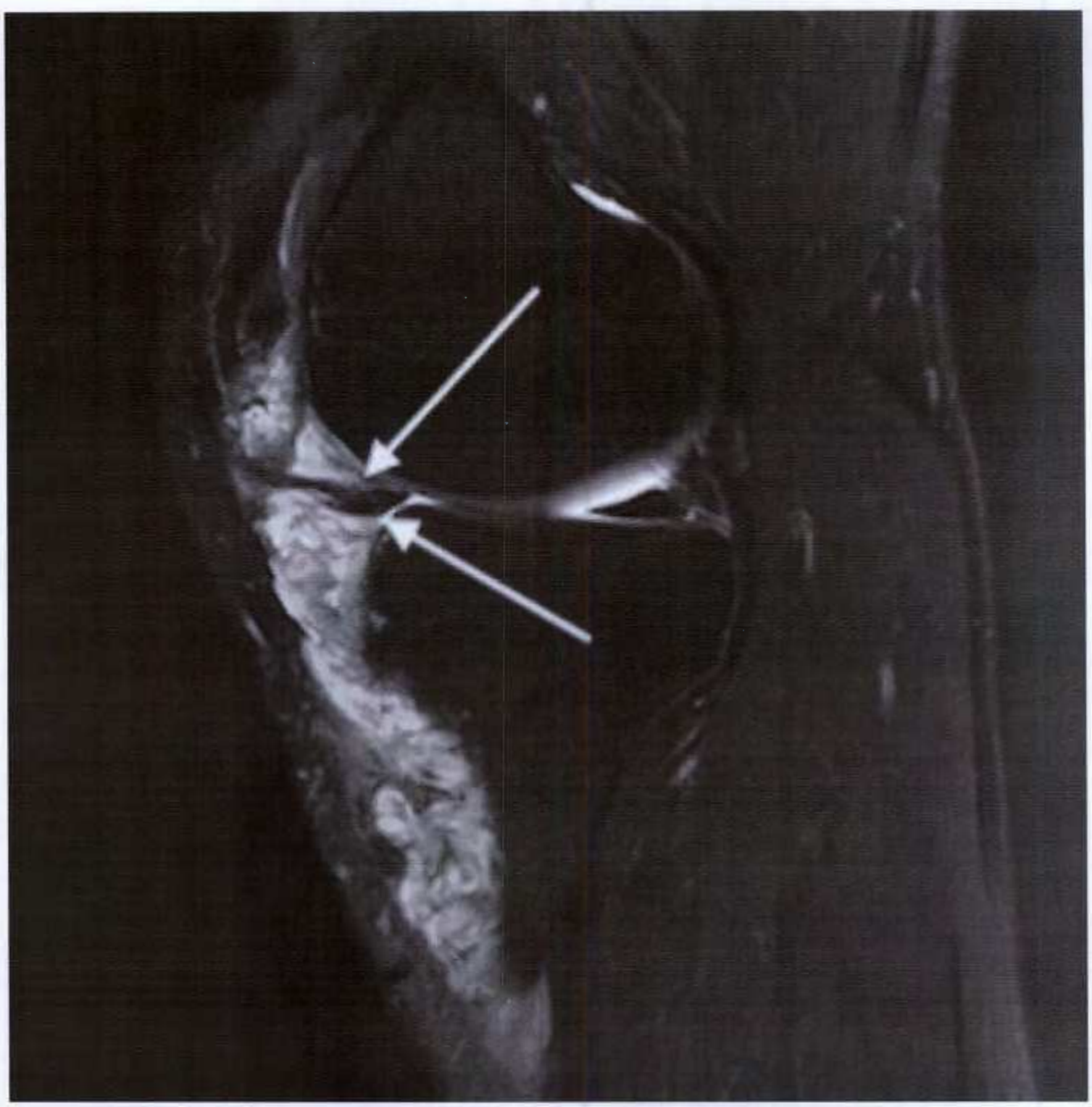




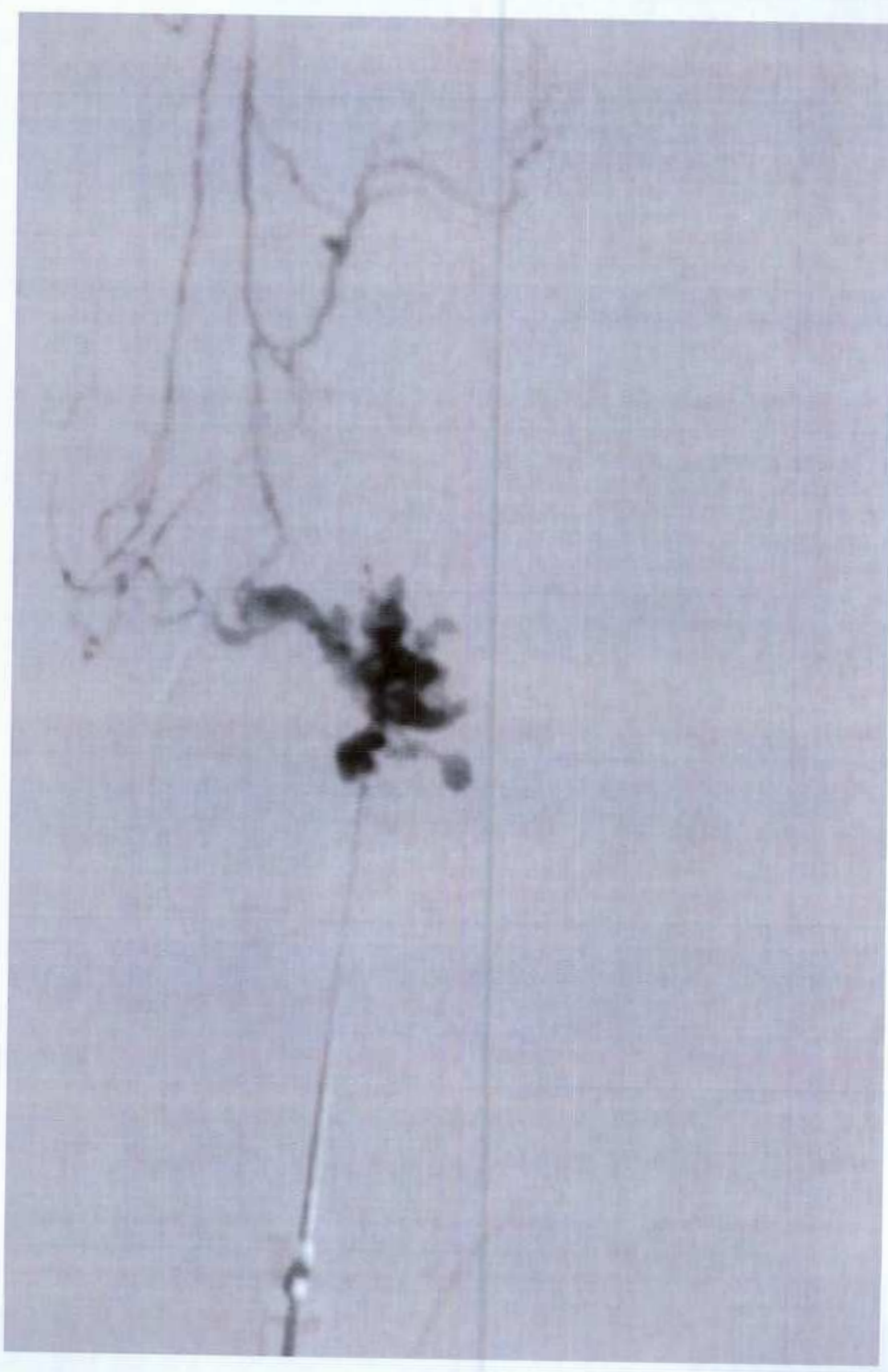


Figure 2c

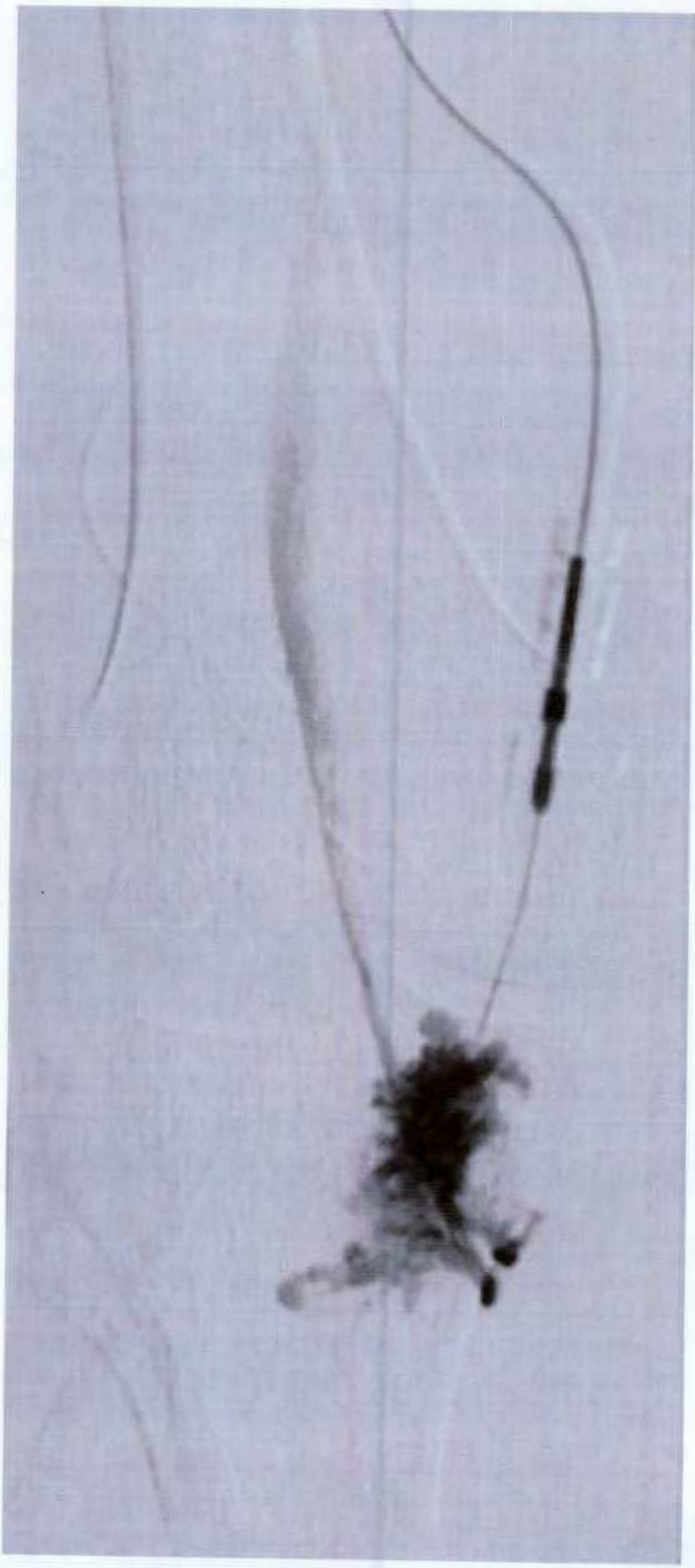




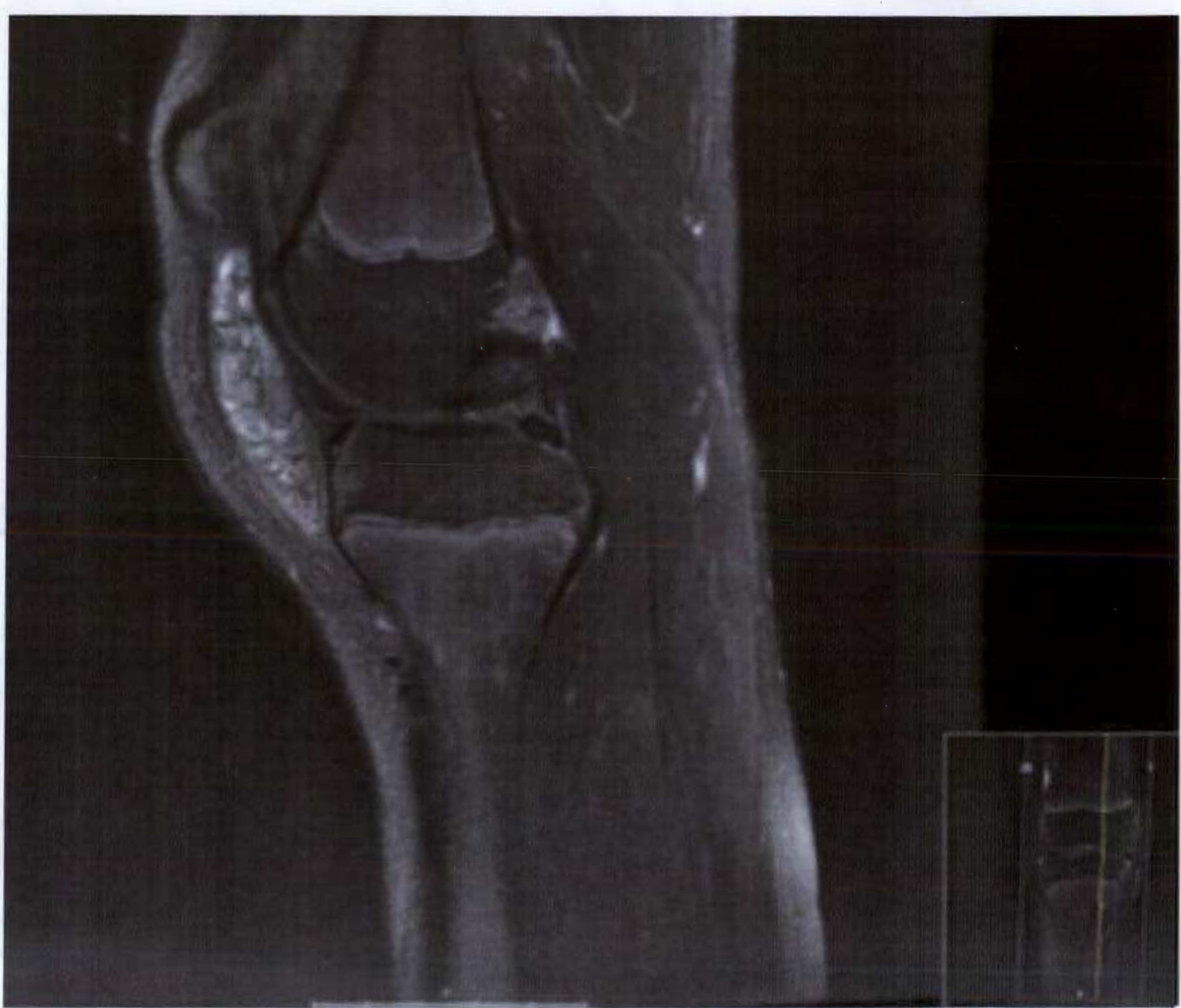




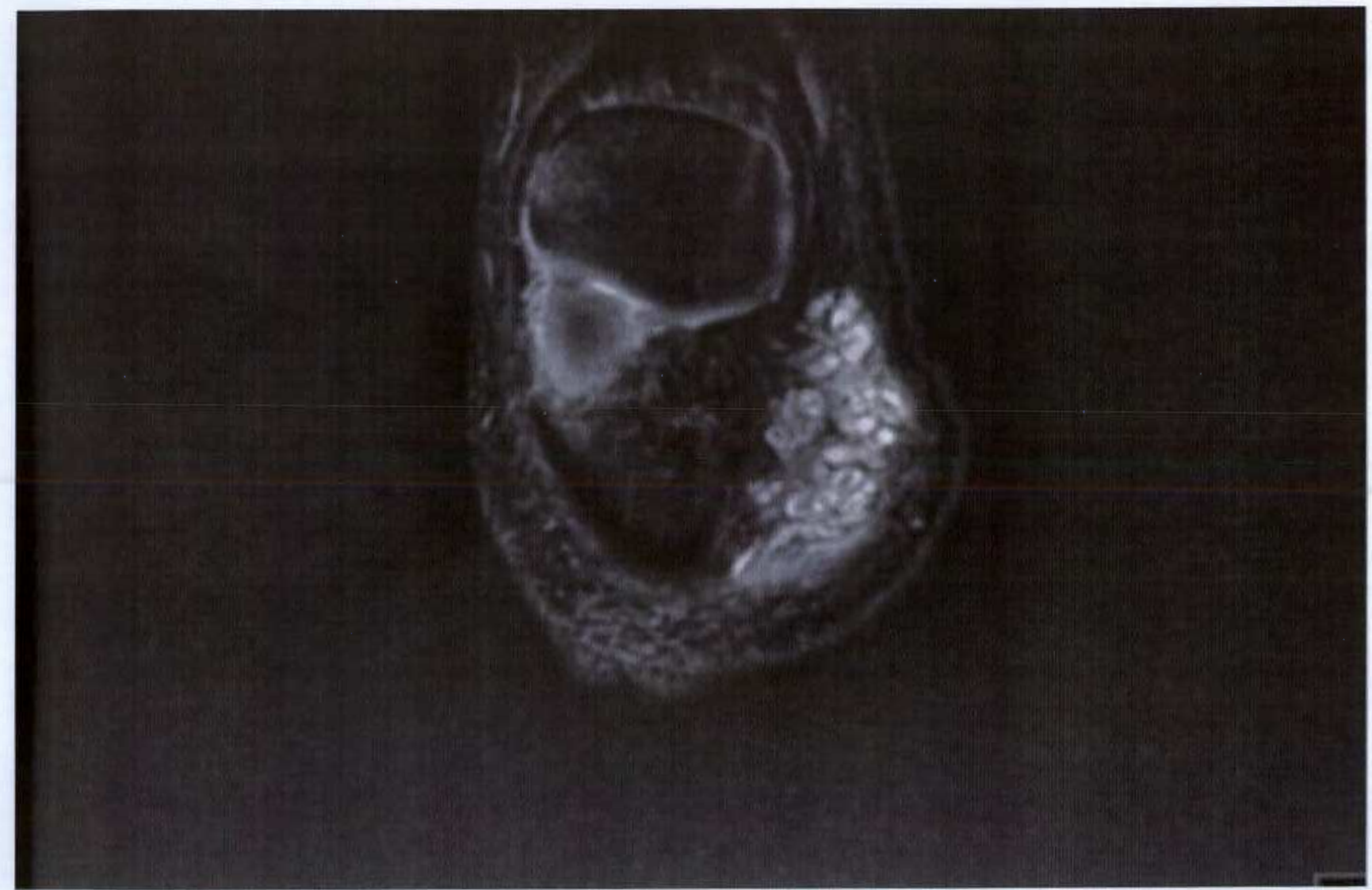


Figure $3 c$

Click here to access/download;Figure;Figure 3c.tiff 1 


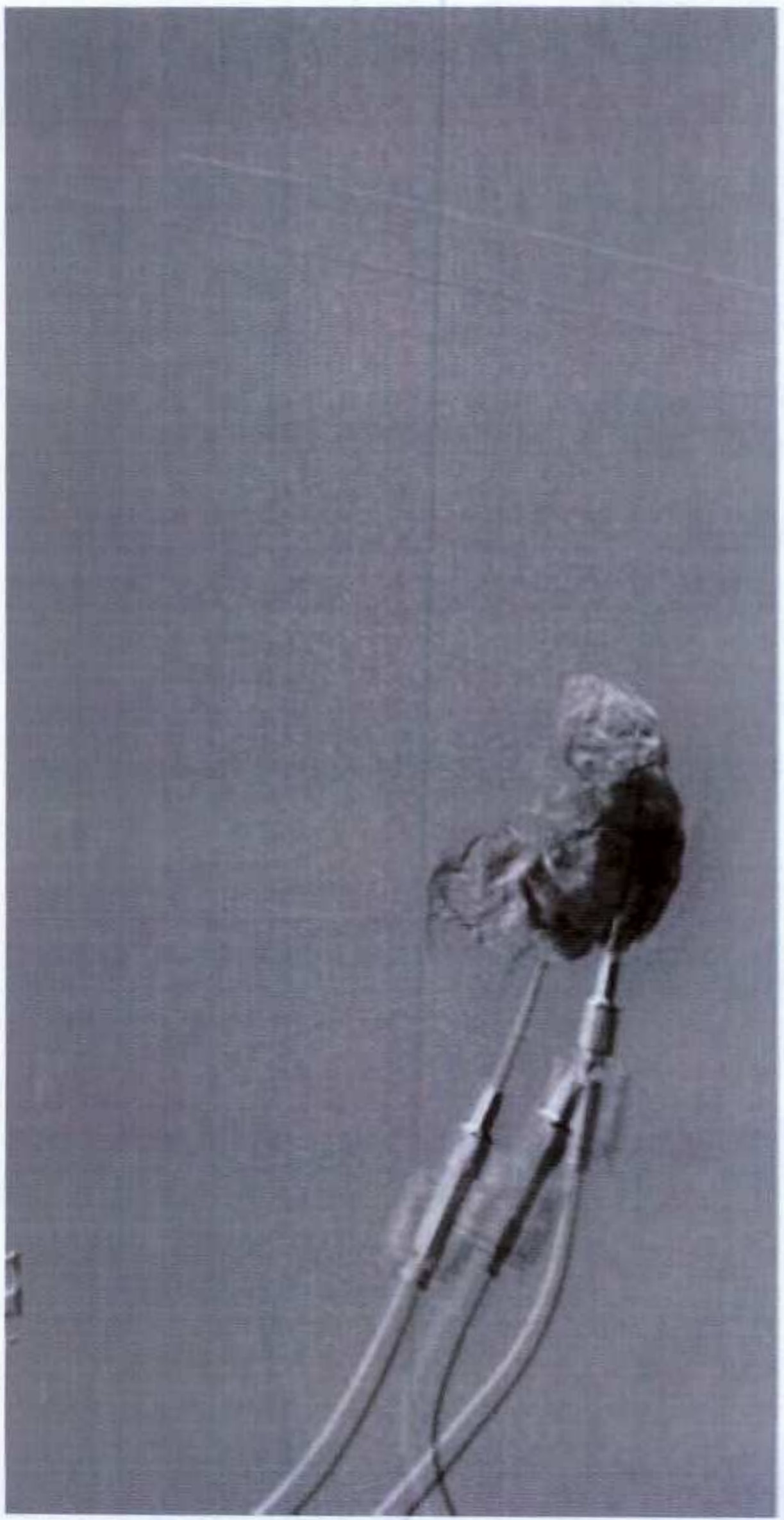




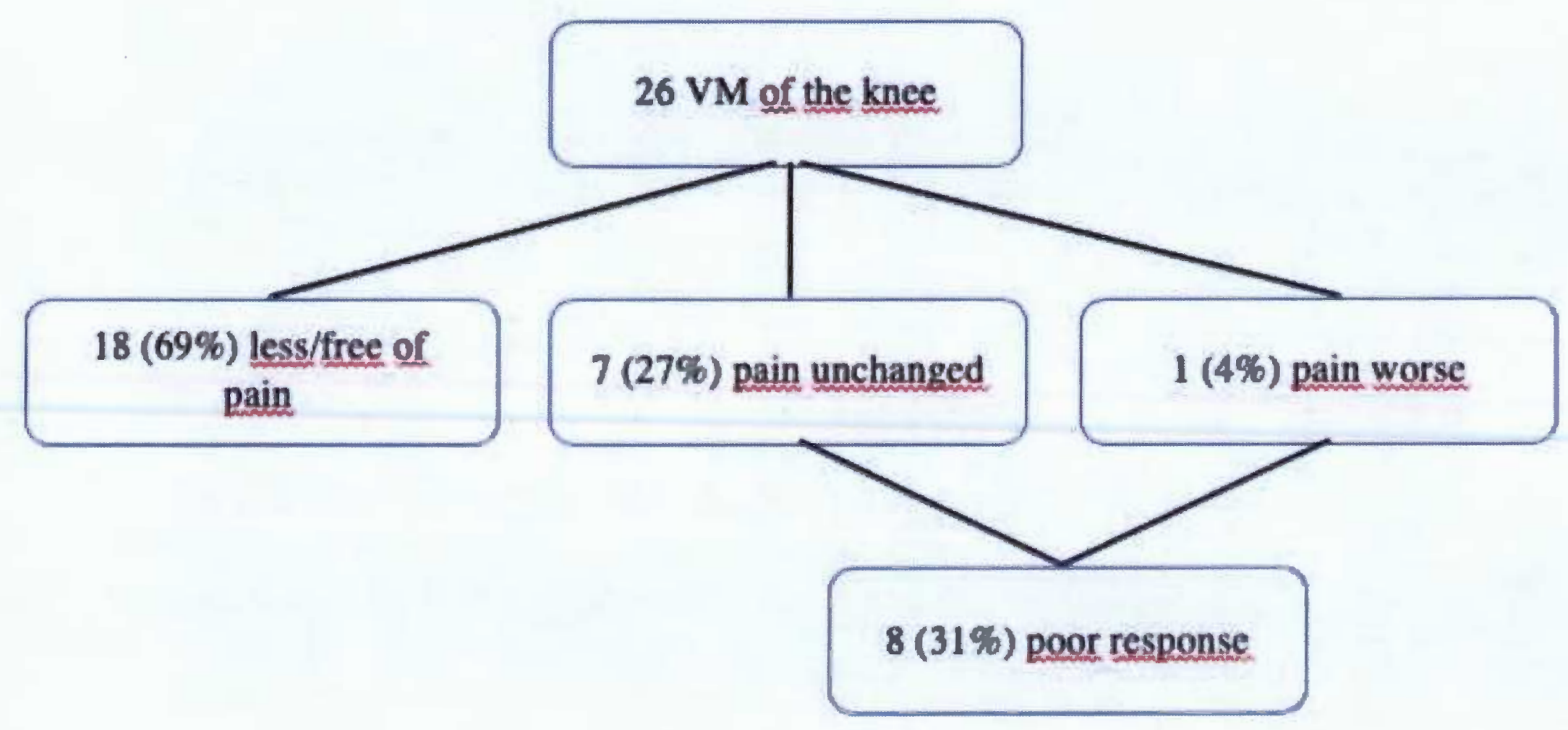

